\title{
TRITERPENÓIDES TIPO CICLOARTANO DE PRÓPOLIS DE TERESINA - PI
}

\author{
Maria do Socorro Sousa da Silva, Antônia Maria das Graças Lopes Citó*, Mariana H. Chaves e José Arimatéia Dantas \\ Lopes \\ Departamento de Química, Universidade Federal do Piauí, 64049-480 Teresina - PI
}

Recebido em 1/7/04; aceito em 16/2/05; publicado na web em 11/7/05

\begin{abstract}
CYCLOARTANE TRITERPENOIDS OF PROPOLIS FROM TERESINA - PI. The chemical study of the propolis produced in Teresina city, state of Piaui, resulted in the identification of six cycloartane triterpenoids: isomangiferolic acid, 24methylenecycloartane-3ß,26-diol, mangiferolic acid, mangiferonic acid, ambonic acid and ambolic acid. The substances were characterized by one and two-dimensional ${ }^{1} \mathrm{H}$ and ${ }^{13} \mathrm{C}$ NMR analysis.
\end{abstract}

Keywords: propolis; triterpenoids; cycloartane.

\section{INTRODUÇÃO}

Própolis é um produto elaborado na colméia a partir de substâncias resinosas, gomosas e balsâmicas, coletadas pelas abelhas de brotos, flores e exsudatos de plantas, acrescidas de secreções salivares, ceras e pólen. A palavra própolis é derivada do grego pro, em defesa de, e polis, a cidade, o que quer dizer "em defesa da cidade ou da colméia" ${ }_{1,2}$. As abelhas utilizam a própolis para protegê-las contra insetos e microrganismos, recobrindo a parede da colméia, reforçando os favos, preenchendo fissuras, restringindo a entrada e embalsamando animais; utilizam-na também no preparo de locais assépticos para a postura da abelha rainha ${ }^{2,3}$.

Existem relatos do uso de própolis em medicina popular desde a antiguidade ${ }^{1}$. No Egito antigo era empregada para embalsamar os mortos. Na África do Sul, na guerra ao final do século XIX, foi amplamente utilizada devido às suas propriedades cicatrizantes e na Segunda Guerra Mundial foi empregada em várias clínicas soviéticas, como base de uma pomada para tratar ferimentos, apresentando excelentes resultados ${ }^{3-5}$. Atualmente, é bastante usada em alimentos e beberagens para manter a saúde e prevenir doenças coronárias, diabetes e até mesmo câncer ${ }^{6}$. A própolis e seus constituintes químicos apresentam várias atividades farmacológicas, tais como antioxidante ${ }^{7}$, antiinflamatória ${ }^{8}$, antibacteriana ${ }^{9}$, antiviral $^{10}$, antifúngica $^{11}$ e antitumoral ${ }^{12}$.

A própolis apresenta em sua composição vários tipos de substâncias, entre as quais triterpenóides, flavonóides, ácidos aromáticos, ácidos graxos, fenóis, aminoácidos, vitaminas $\mathrm{A}, \mathrm{B}_{1}, \mathrm{~B}_{2}, \mathrm{~B}_{6}, \mathrm{C}$ e E e minerais, como $\mathrm{Mn}, \mathrm{Cu}, \mathrm{Ca}, \mathrm{Al}, \mathrm{V}, \mathrm{Ni}, \mathrm{Zn}$ e $\mathrm{Cr}$. A proporção dessas substâncias varia e depende do local e da época da coleta ${ }^{1,12}$. Pesquisas mostraram que a composição da própolis é muito similar à dos exsudatos de brotos das plantas visitadas pelas abelhas ${ }^{13}$. A própolis brasileira apresenta em sua composição compostos aromáticos ${ }^{14} \mathrm{e}$, recentemente, têm sido relatados triterpenóides ${ }^{15}$. Essas substâncias apresentam diversas atividades farmacológicas, tais como antiinflamatória ${ }^{16,17}$, antiviral ${ }^{15,16} \mathrm{e}$ antitumoral ${ }^{16}$.

Devido aos diversos usos da própolis e de sua complexa composição, que varia de acordo com a flora apícola da região de sua produção, vários estudos sobre os constituintes químicos e a ação farmacológica têm sido realizados para indicar seu real potencial como produto terapêutico.

*e-mail: gracito@ufpi.br
O estado do Piauí apresenta um forte potencial apícola, mas sua pesquisa nesta área ainda é incipiente; daí surgiu nosso interesse em estudar a composição química da própolis piauiense. Em estudos anteriores sobre a própolis piauiense foi relatada a presença de derivados de ácido anacárdico, triterpenóides pentacíclicos e derivados do cicloartano ${ }^{18}$. O presente trabalho relata o estudo de três amostras de própolis produzidas em diferentes épocas na cidade de Teresina - PI, nas quais foram identificados seis triterpenóides derivados do cicloartano: ácido isomangiferólico (1), ácido mangiferólico (2), ácido mangiferônico (3), 24-metilenocicloartano-3ß,26-diol (4), ácido ambólico (5) e ácido ambônico (6) inéditos em própolis. Triterpenóides com esqueletos $\mathrm{C}_{30}$ e $\mathrm{C}_{31}$, baseados no cicloartano, são característicos da espécie Mangifera indica (Anacardiaceae) ${ }^{19}$.

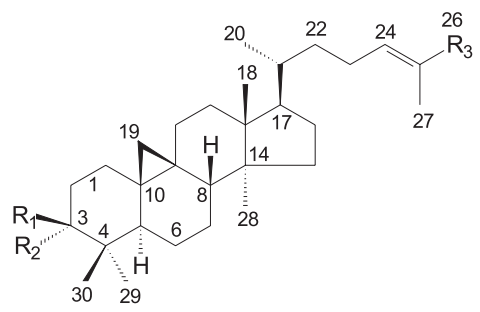

$$
\begin{aligned}
& 1 \mathrm{R}_{1}=\mathrm{H}, \mathrm{R}_{2}=\mathrm{OH}, \mathrm{R}_{3}=\mathrm{COOH} \\
& 2 \mathrm{R}_{1}=\mathrm{OH}, \mathrm{R}_{2}=\mathrm{H}, \mathrm{R}_{3}=\mathrm{COOH} \\
& 3 \mathrm{R}_{1}, \mathrm{R}_{2}=\mathrm{O}, \mathrm{R}_{3}=\mathrm{COOH}
\end{aligned}
$$

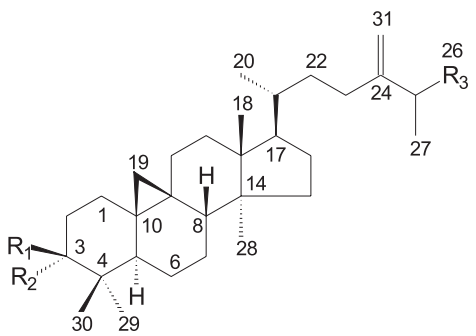

$$
\begin{aligned}
& 4 \mathrm{R}_{1}=\mathrm{OH}, \mathrm{R}_{2}=\mathrm{H}, \mathrm{R}_{3}=\mathrm{CH}_{2} \mathrm{OH} \\
& 5 \mathrm{R}_{1}=\mathrm{OH}, \mathrm{R}_{2}=\mathrm{H}, \mathrm{R}_{3}=\mathrm{COOH} \\
& 6 \mathrm{R}_{1}, \mathrm{R}_{2}=\mathrm{O}, \mathrm{R}_{3}=\mathrm{COOH}
\end{aligned}
$$

Figura 1. Triterpenóides tipo cicloartano identificados em própolis de Teresina-PI 


\section{PARTE EXPERIMENTAL}

\section{Procedimentos experimentais gerais}

Os espectros de $\mathrm{RMN}{ }^{1} \mathrm{H}$ e ${ }^{13} \mathrm{C}$ foram registrados em espectrômetros Brüker modelos Avance DRX-500 e Avance DPX300. Os deslocamentos químicos foram registrados em $\delta$ (ppm), utilizando-se $\mathrm{CDCl}_{3}$ como solvente e TMS como padrão interno.

As análises em CCD foram realizadas em cromatoplacas de gel de sílica, constituídas de uma mistura na proporção 1:1 de gel de sílica $\mathrm{G} 60$ e gel de sílica $\mathrm{GF}_{254}$. As cromatoplacas foram reveladas através de irradiação com luz ultravioleta em comprimentos de onda de 254 e $366 \mathrm{~nm}$ em boxe da CAMAG e/ou aquecimento em uma placa elétrica, após borrifação com uma solução de sulfato cérico, $\left[\mathrm{Ce}\left(\mathrm{SO}_{4}\right)_{2} .5 \mathrm{H}_{2} \mathrm{O}\right]^{20}$. Para as colunas cromatográficas utilizou-se gel de sílica com granulometria de 0,063 a $0,20 \mathrm{~mm}$. As colunas em Sephadex LH-20 foram antecedidas de purificação do gel mediante aquecimento em metanol, seguido de filtração a vácuo e secagem em estufa a $55-60{ }^{\circ} \mathrm{C}$ por $12-16 \mathrm{~h}$. Os eluentes utilizados nas colunas em Sephadex LH-20 foram hexano- $\mathrm{CH}_{2} \mathrm{Cl}_{2}$ (1:4), $\mathrm{CH}_{2} \mathrm{Cl}_{2}$-acetona $(3: 2)$ e $\mathrm{CH}_{2} \mathrm{Cl}_{2}$-acetona $(1: 4)^{21}$.

\section{Coleta das amostras}

As amostras de própolis da espécie Apis mellifera foram coletadas no apiário do Departamento de Zootecnia do Centro de Ciências Agrárias da Universidade Federal do Piauí, em Teresina, nos meses de abril, julho e setembro de 2002, acondicionadas em recipientes de vidro e mantidos sob refrigeração. Estas amostras foram denominadas $\mathrm{A}, \mathrm{B}$ e $\mathrm{C}$, respectivamente.

\section{Extração e isolamento dos constituintes químicos}

As amostras de própolis A (13,3 g), B (16,0 g) e C (16,0 g) foram submetidas à extração com metanol em aparelhagem Soxhlet, por um período de $5 \mathrm{~h}$ e $30 \mathrm{~min}$ (amostras A e B) e por $10 \mathrm{~h}$ (amostra C). Os extratos filtrados a quente foram diluídos com água, na proporção de 2:1, extrato:água e, em seguida, foram efetuadas sucessivas extrações com hexano e éter etílico ${ }^{22}$. Após concentração dos extratos obteve-se as frações hexânicas $\mathrm{A}_{1}(0,6 \mathrm{~g}), \mathrm{B}_{1}(1,7 \mathrm{~g}) \mathrm{e}$ $\mathrm{C}_{1}(1,1 \mathrm{~g})$ e as frações etéreas $\mathrm{A}_{2}(3,5 \mathrm{~g}), \mathrm{B}_{2}(2,5 \mathrm{~g})$ e $\mathrm{C}_{2}(6,9 \mathrm{~g})$. As frações obtidas foram analisadas por $\mathrm{CCD}$, sendo as frações $\mathrm{A}_{2}, \mathrm{~B}_{2}$ e $\mathrm{C}_{2}$ escolhidas para análises dos constituintes químicos.

$\mathrm{A}$ fração $\mathrm{A}_{2}$ foi submetida à $\mathrm{CC}$ de gel de sílica, utilizando-se um gradiente de eluição de polaridade crescente com hexano e éter etílico, fornecendo 65 sub-frações. A sub-fração $\mathrm{A}_{2}-27$ (134 mg), eluída com hexano:éter etílico (2:1), foi recromatografada em coluna de Sephadex LH-20, fornecendo 33 sub-frações. A sub-fração $\mathrm{A}_{2}$-27-28 (3 mg), eluída com diclorometano:acetona (3:2), foi identificada como ácido isomangiferólico (1).

A fração $B_{2}$ foi fracionada por $\mathrm{CC}$ de gel de sílica, eluída com hexano e acetato de etila em um gradiente de eluição de polaridade crescente, originando 100 sub-frações. Na sub-fração $\mathrm{B}_{2}$-27 (134 $\mathrm{mg}$ ), eluída com hexano:acetato de etila (95:5), foi identificada a presença do 24-metileno-cicloartano-3 $\beta, 26$-diol (4) e na sub-fração $B_{2}-46$ (11 mg), eluída com hexano:acetato de etila (8:2), o ácido mangiferólico (2).

$\mathrm{A}$ fração $\mathrm{C}_{2}$ foi submetida à $\mathrm{CC}$ de gel de sílica, eluída com hexano e acetato de etila em um gradiente de eluição de polaridade crescente, resultando em 107 sub-frações. A sub-fração $\mathrm{C}_{2}-54$ (151 mg) eluída com hexano:acetato de etila (8:2), e a sub-fração $C_{2}-70$ (150 mg), eluída com hexano:acetato de etila (7:3), foram recromatogradas em coluna de Sephadex LH-20 e forneceram 32 sub-frações cada. As sub-frações $\mathrm{C}_{2}-54-24$ (13 mg) e $\mathrm{C}_{2}-70-23$ (16 mg) foram eluídas com diclorometano:acetona (3:2), sendo a primeira constituída pelos ácidos mangiferônico (3) e ambônico (6) e a segunda, pelo ácido ambólico (5).

\section{RESULTADOS E DISCUSSÃO}

As análises preliminares em CCD das sub-frações, resultantes dos fracionamentos cromatográficos das frações etéreas de própolis, mostraram que algumas destas apresentavam natureza terpênica evidenciada pela coloração roxa, obtida após revelação das cromatoplacas com sulfato cérico.

A identificação dos triterpenóides foi realizada através da análise dos espectros de $\mathrm{RMN}{ }^{1} \mathrm{H},{ }^{13} \mathrm{C}$ e DEPT $135^{\circ}$ e foi feita a comparação com dados da literatura. Em alguns casos, utilizou-se, também, os espectros de COSY ${ }^{1} \mathrm{H}-{ }^{1} \mathrm{H}$, HMQC e HMBC. Os deslocamentos químicos dos carbonos dos triterpenóides identificados neste trabalho estão listados na Tabela 1.

As frações em éter etílico, obtidas das partições dos extratos metanólicos de própolis, forneceram após fracionamentos em coluna de gel de sílica e Sephadex LH-20 seis triterpenóides: ácido isomangiferólico (1), ácido mangiferólico (2), ácido mangiferônico (3), 24-metileno-cicloartano-3ß,26-diol (4), ácido ambólico (5) e ácido ambônico (6). Estes triterpenóides possuem como esqueleto base o cicloartano, são característicos da espécie Mangifera indi$c a^{19,23-26}$ e inéditos em própolis.

O triterpenóide 1 apresenta em seu espectro de $\mathrm{RMN}{ }^{1} \mathrm{H}$ dois dubletos blindados, em $\delta 0,37(\mathrm{~J}=4,0 \mathrm{~Hz})$ e $\delta 0,55(\mathrm{~J}=4,0 \mathrm{~Hz})$, ca-

Tabela 1. Dados de $\mathrm{RMN}{ }^{13} \mathrm{C}$ de 1 a $6\left[\mathrm{CDCl}_{3}, * 125\right.$ e **75 MHz]

\begin{tabular}{|c|c|c|c|c|c|c|}
\hline $\mathrm{C}$ & $1^{*}$ & $2 *$ & $3 * *$ & $4 * *$ & $5 * *$ & $6 * *$ \\
\hline 1 & 27,7 & 31,9 & 33,4 & 31,9 & 31,9 & 33,4 \\
\hline 2 & 28,8 & 30,3 & 37,4 & 30,3 & 30,3 & 37,4 \\
\hline 3 & 77,4 & 78,8 & 216,7 & 78,9 & 78,9 & 216,7 \\
\hline 4 & 39,8 & 40,5 & 50,2 & 40,5 & 40,4 & 50,2 \\
\hline 5 & 41,3 & 47,1 & 48,4 & 47,1 & 47,1 & 48,4 \\
\hline 6 & 21,3 & 21,1 & 21,5 & 21,1 & 21,1 & 21,5 \\
\hline 7 & 25,8 & 26,4 & 28,1 & 28,2 & 28,1 & 28,1 \\
\hline 8 & 48,3 & 47,9 & 47,9 & 47,8 & 48,0 & 47,9 \\
\hline 9 & 20,0 & 19,9 & 21,1 & 20,0 & 20,0 & 21,1 \\
\hline 10 & 26,7 & 26,1 & 25,9 & 26,1 & 26,0 & 25,9 \\
\hline 11 & 26,5 & 25,9 & 25,9 & 26,0 & 26,1 & 25,9 \\
\hline 12 & 33,1 & 32,9 & 32,8 & 32,9 & 32,9 & 32,8 \\
\hline 13 & 45,5 & 45,3 & 45,5 & 45,3 & 45,3 & 45,4 \\
\hline 14 & 49,1 & 48,8 & 48,7 & 48,8 & 48,8 & 48,7 \\
\hline 15 & 35,7 & 35,5 & 35,5 & 35,5 & 35,5 & 35,5 \\
\hline 16 & 28,4 & 28,1 & 26,9 & 26,5 & 26,5 & 26,9 \\
\hline 17 & 52,4 & 52,2 & 52,2 & 52,2 & 52,2 & 52,3 \\
\hline 18 & 18,3 & 18,0 & 18,1 & 19,3 & 19,3 & 18,1 \\
\hline 19 & 30,2 & 29,9 & 29,5 & 29,9 & 29,9 & 29,5 \\
\hline 20 & 36,2 & 35,9 & 35,9 & 36,1 & 36,0 & 36,0 \\
\hline 21 & 18,2 & 18,1 & 18,3 & 18,0 & 18,3 & 18,3 \\
\hline 22 & 35,0 & 34,8 & 34,7 & 34,9 & 34,5 & 34,7 \\
\hline 23 & 26,1 & 25,9 & 25,9 & 31,2 & 31,6 & 31,6 \\
\hline 24 & 145,9 & 145,7 & 145,8 & 152,0 & 148,6 & 148,6 \\
\hline 25 & 126,6 & 126,6 & 126,5 & 36,1 & 45,5 & 45,6 \\
\hline 26 & 172,1 & 173,0 & 173,0 & 65,9 & 179,2 & 179,0 \\
\hline 27 & 12,2 & 11,9 & 11,9 & 16,4 & 16,3 & 16,3 \\
\hline 28 & 19,5 & 19,3 & 18,1 & 18,3 & 18,0 & 18,3 \\
\hline 29 & 21,4 & 25,4 & 22,2 & 25,5 & 25,4 & 22,2 \\
\hline 30 & 26,1 & 14,0 & 20,8 & 14,0 & 14,0 & 20,8 \\
\hline 31 & - & - & - & 109,5 & 111,1 & 111,1 \\
\hline
\end{tabular}


racterísticos de hidrogênios metilênicos de ciclopropano; apresenta também um dubleto em $\delta 0,94(\mathrm{~J}=6,6 \mathrm{~Hz})$ e cinco singletos em $\delta$ 0,$91 ; 0,93 ; 0,98 ; 0,99$ e 1,88, atribuídos a hidrogênios de grupos metilícos. $\mathrm{O}$ espectro de $\mathrm{RMN}{ }^{1} \mathrm{H}$ apresenta ainda um singleto largo em $\delta 3,49$, cuja aparência e deslocamento químico são característicos em triterpenóides, de H-3 em carbono cabinólico com configuração $\beta$-equatorial indicando a presença de um triterpenóide tipo cicloartano-3 $\alpha-\mathrm{ol}^{25,27}$. Em contraste, se a configuração de H-3 fosse $\alpha$-axial $(\mathrm{OH} \beta$-equatorial) o sinal seria um duplo dubleto em aproximadamente $\delta 3,2$ com $\mathrm{J}_{\mathrm{ax}-\mathrm{ax}}=11 \mathrm{~Hz}$ e $\mathrm{J}_{\mathrm{ax}-\mathrm{eq}}=4,2 \mathrm{~Hz}^{28,29}$. O tripleto em $\delta 6,92(\mathrm{~J}=7,0 \mathrm{~Hz})$ refere-se a hidrogênio olefínico em ligação dupla com configuração $E$ e em posição $\beta$ a um grupo retirador de elétrons ${ }^{23,25,30}$. $\mathrm{O}$ espectro de $\mathrm{RMN}{ }^{13} \mathrm{C}$ apresenta vinte e nove sinais, entre os quais encontram-se um grupo oximetínico em $\delta 77,4$ (C-3), que é também indicativo de configuração $3 \alpha$ hidroxi $^{25,31}$, uma carboxila $\alpha, \beta$-insaturada em $\delta 172,1$ (C-26) e dois carbonos olefínicos, em $\delta$ 126,6 (C-25) e 145,9 (C-24). A localização da carboxila em C-26 foi atribuída à existência de um carbono metílico em $\delta$ 12,2 (C-27), bastante blindado, decorrente da interação deste com $\mathrm{C}-23^{25}$, reforçando a sugestão da configuração $E$ para a ligação dupla. Pelo espectro de HMBC observaram-se as correlações do hidrogênio olefínico com C-27 e C-26, bem como dos hidrogênios metílicos do C-27 com C-24, C-25 e C-26, confirmando a posição da carboxila na cadeia lateral do triterpenóide. À exceção de C-7, C-16 e C-23, todos os dados de RMN ${ }^{13} \mathrm{C}$ da substância 1 estão consistentes com os registrados na literatura para o ácido isomangiferólico ${ }^{25,27}$. Os valores de C-7 e C-16 apresentamse invertidos em relação aos da literatura ${ }^{25}$; isto foi comprovado através das análises no $\operatorname{COSY}{ }^{1} \mathrm{H}-{ }^{1} \mathrm{H}, \mathrm{HMBC}$ e HMQC. No HMBC observam-se as correlações entre os sinais em $\delta 1,32(\mathrm{H}-16)$ com $\delta$ 35,7 (C-15) e de $\delta 1,18$ (H-7) com $\delta 21,3$ (C-6); no $\operatorname{COSY}{ }^{1} \mathrm{H}-{ }^{1} \mathrm{H}$ o sinal em $\delta 0,82$ (H-6) correlaciona-se com $\delta 1,18$ (H-7), comprovando-se a permuta entre os valores dos deslocamentos de C-7 e C-16. A análise dos espectros RMN ${ }^{13} \mathrm{C}-\mathrm{BB}$, HMQC e HMBC possibilitou uma reatribuição do valor de C-23, que na literatura apresenta um valor de $\delta 21,0^{25}$ para $\delta 26,1$; no HMQC observa-se que o sinal em $\delta 26,1\left(\mathrm{CH}_{2}\right)$ se correlaciona com os hidrogênios em $\delta$ 2,16 e $\delta 2,28$ (H-23) e no HMBC, estes se correlacionam com o sinal de $\delta$ 145,9 (C-24), comprovando que o sinal em $\delta$ 26,1 é relativo a $\mathrm{C}-23$.

O espectro de $\mathrm{RMN}{ }^{1} \mathrm{H}$ de $\mathbf{2}$, semelhante a $\mathbf{1}$, apresenta sinais de triterpenóides derivados do cicloartano, sendo que o sinal de $\mathrm{H}$ 3 , em 2, é um duplo dubleto em $\delta 3,30(\mathrm{~J}=4,4$ e $11,1 \mathrm{~Hz})$ característico de hidrogênio $\alpha$-axial em triterpenóides $3 \beta-\mathrm{OH}^{28,29,31}$, conforme discutido para a elucidação da substância $\mathbf{1}$. $\mathrm{O}$ espectro de $\mathrm{RMN}{ }^{13} \mathrm{C}$ possui trinta sinais, incluindo um grupo oximetino em $\delta$ 78,8 (C-3) consistente com a configuração $3 \beta$-hidroxi ${ }^{25,31}$, uma carboxila em $\delta 173,0$ (C-26) e dois carbonos olefínicos, em $\delta$ 126,6 (C-25) e $\delta$ 145,7(C-24). Observou-se para a substância 2 que os carbonos C-1 a C-5, C-29 e C-30 apresentam uma diferença em seus deslocamentos químicos se comparados aos do ácido isomangiferólico (1) e semelhantes aos do cicloartenol ${ }^{25}$, confirmando a configuração $3 \beta$-hidroxi. A posição da carboxila em C-26 e a estereoquímica da ligação dupla, $E$, foram confirmadas pelo deslocamento de C-27 ( $\delta 11,9)$. Os dados observados para a substância 2 estão coerentes com os existentes na literatura para o ácido mangiferólico $(\mathbf{2})^{19,23}$.

No espectro de RMN ${ }^{1} \mathrm{H}$ do triterpenóide 5 observaram-se sinais característicos de triterpenóide com esqueleto cicloartano, juntamente com um duplo-dubleto em $\delta 3,30(\mathrm{~J}=4,4$ e $11 \mathrm{~Hz})$, característicos de uma configuração $\beta$-hidroxi, um quarteto em $\delta 3,17$ $(\mathrm{J}=7,1 \mathrm{~Hz})$ e dois singletos largos em $\delta 4,93$ e 4,97, referentes a hidrogênios metilênicos em ligação dupla geminal dissubstituída.
$\mathrm{O}$ espectro de $\mathrm{RMN}{ }^{13} \mathrm{C}$ apresenta trinta e um sinais, entre eles de um grupo oximetino em $\delta 78,9$ (C-3) que confirma a configuração $3 \beta$-hidroxi ${ }^{29}$, dois de carbonos olefínicos em $\delta 111,1$ (C-31) e $\delta$ 148,6 (C-24) e um de carboxila em $\delta$ 179,2 (C-26). Estes dados são compatíveis com um triterpenóide tipo cicloartano-3 $\beta$-ol, que possui um grupo metilênico e uma carboxila ligados a sua cadeia late$\mathrm{ral}^{26}$. No espectro de HMBC observaram-se as correlações dos hidrogênios olefínicos $(\delta 4,93$ e 4,97) com C-23 e C-25 e do quarteto em $\delta 3,17$, atribuído ao hidrogênio de C-25 com C-24 e C-26. Estes dados confirmam a localização do grupo metilênico sobre C-24 e da carboxila em C-26. Os dados da substância 5 estão consistentes com os citados na literatura para o ácido ambólico ${ }^{19,26}$.

A análise dos espectros de RMN da sub-fração $B_{2}-29$ mostrou que esta possui uma mistura de substâncias, da qual foram identificadas apenas o triterpenóide 4. O espectro de RMN ${ }^{1} \mathrm{H}$ da sub-fração $B_{2}-29$ apresenta, para o constituinte majoritário, sinais característicos de um triterpenóide tipo cicloartano-3 $\beta$-ol. Além destes, apresenta um sinal em $\delta 3,58$, referente a hidrogênios ligados a um segundo carbono carbinólico e dois singletos de hidrogênios olefínicos, em $\delta 4,88$ e 4,94. Estes dados são compatíveis com um triterpenóide tipo cicloartano-3 $\beta$-ol ${ }^{28,29}$, com a cadeia lateral com uma ligação dupla geminal dissubstituída e uma hidroxila em carbono primário. $\mathrm{O}$ espectro de $\mathrm{RMN}{ }^{13} \mathrm{C}$ apresenta sinais de um

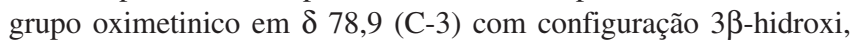
um grupo oximetilênico em $\delta 65,9(\mathrm{C}-26)$ e dois carbonos olefínicos em $\delta 109,5(\mathrm{C}-31)$ e $\delta 152,0$ (C-24) na cadeia lateral do triterpenóide $^{24,26}$. Os dados da substância 4 são equivalentes aos citados na literatura para o 24-metileno-cicloartano-3 $\beta, 26-$ diol $^{26}$.

A mistura de substâncias da sub-fração $\mathrm{C}_{2}-54-25$ apresenta em seu espectro de $\mathrm{RMN}{ }^{1} \mathrm{H}$ sinais que indicam a presença de triterpenóide tipo cicloartano-3-oxo, pois não apresentou sinal de hidrogênio em carbono carbinólico ${ }^{29,32}$. Observou-se, também, um tripleto em $\delta 6,92(\mathrm{~J}=7,0 \mathrm{~Hz})$, que sugere a existência de um hidrogênio olefínico $\beta$-carboxílico em uma ligação dupla com configuração $E^{23,25,30}$, caracterizando 3. Um quarteto em $\delta 3,18(\mathrm{~J}=7,0 \mathrm{~Hz})$ e dois singletos largos em $\delta$ 4,94 e 4,98 de hidrogênios metilênicos em ligação dupla terminal caracterizam a substância 6. O espectro de $\mathrm{RMN}{ }^{13} \mathrm{C}$ apresenta vários sinais, destacando-se um sinal referente às carbonilas dos dois triterpenóides em $\delta$ 216,7 (C-3), dois carbonos olefínicos em $\delta 145,8(\mathrm{C}-24)$ e $\delta 126,5$ (C-25) referentes à substância 3, dois carbonos olefínicos em $\delta 111,1$ (C-31) e $\delta$ 148,6 (C-24) da 6. Estes dados foram compatíveis com os observados para os ácidos mangiferônico ${ }^{19,26}(\mathbf{3})$ e ambônico ${ }^{26}(\mathbf{6})$. Para a substância 3, da mesma forma que em 1, foi atribuído a C-23 um novo valor para seu deslocamento químico. Na literatura consta o valor $\delta 22,2^{26}$, mas o espectro de $\mathrm{RMN}{ }^{13} \mathrm{C}$ da mistura de $\mathbf{3}$ e $\mathbf{6}$ não apresenta este sinal e, após a atribuição dos deslocamentos químicos dos demais carbonos, restou um sinal de $\mathrm{CH}_{2}$ em $\delta 25,9$, que foi atribuído a C-23.

Triterpenóides pentacíclicos tipo lupeol, $\alpha$ e $\beta$-amirinas e tetracíciclicos tipo cicloartenol e 24-metilenocicloartanol apresentam atividade antiinflamatória significativa ${ }^{16,17}$. O isolamento deste tipo de substância em própolis piauiense poderá contribuir para validação do seu uso como antitinflamatório.

Os estudos realizados mostram que existe uma correlação entre as diversas substâncias presentes na própolis e local e época da sua coleta. Os seis triterpenóides derivados do cicloartano, ácido isomangiferólico (1), ácido mangiferólico (2), ácido mangiferônico (3), 24-metileno-cicloartano-3ß,26-diol (4), ácido ambólico (5) e ácido ambônico (6), relatados neste trabalho foram anteriormente isolados em cascas do caule de Mangifera indica, não existindo relatos na literatura sobre a identificação destes em própolis. Considerando a presença destas substâncias nas amostras da própolis 
em estudo e o fato que a própolis apresenta uma composição similar à dos exsudatos de brotos de plantas visitadas pelas abelhas, podemos propor que a espécie Mangifera indica foi utilizada pelas abelhas, em diferentes épocas do ano, para coleta de materiais para elaboração da própolis estudada e que houve um transporte direto destas substâncias, pelas abelhas, para a colméia. Vale ressaltar que o apiário em estudo tem um mangueiral em sua vizinhança.

\section{AGRADECIMENTOS}

À CAPES pela bolsa de Mestrado (M. S. S. Silva) e apoio financeiro (PROCAD No 0014/01-0), ao Prof. D. C. Souza e sua equipe pelo fornecimento das amostras de própolis, ao CENAUREM/UFC e à Profa. N. V. Gramosa pela obtenção dos espectros de RMN.

\section{REFERÊNCIAS}

1. Ghisalbert, E. L.; Bee World 1979, 60, 59.

2. Marcucci, M. C.; Apidologie 1995, 26, 83.

3. Marcucci, M. C.; Quim. Nova 1996, 19, 529.

4. Ioirish, N.; As Abelhas: Farmacêuticas com Asas, Editora Mir: Moscou, 1982.

5. Pereira, A. S.; Seixas, F. R. M. S.; Aquino Neto, F. R.; Quim. Nova 2002, 25,321 .

6. Banskota, A. H.; Tezuka, Y.; Adnyana, I. K.; Midorikawa, K.; Matsushige, K.; Message, D.; Huertas, A. A. G.; Kadota, S.; J. Etnopharmacol. 2000, 72, 239.

7. Isla, M. I.; Nieva Moreno, M. I.; Sampietro, A. R.; Vattuone, M. A.; J. Etnopharmacol. 2001, 76, 165.

8. Dobrowolski, J. W.; Vohora, S. B.; Sharma, K.; Shah, S. A.; Naqvi, S. A. H.; Dandiya, P. C.; J. Etnopharmacol. 1991, 35, 77.

9. Bankova, V.; Christov, R.; Kujumgiev, A.; Marcucci, M. C.; Popov, S.; Z. Naturforsch. 1995, 50, 167.

10. Amoros, M.; Sauvager, F.; Girre, L.; Cormier, M.; Apidologie 1992, 23, 231.

11. Murad, J. M.; Calvi, S. A.; Soares, A. M. V. C.; Bankova, V.; Sforcin, J. M.; J. Etnopharmacol. 2002, 79, 331.
12. Grunberger, D.; Banerjee, R; Eisinger, K.; Oltz, E. M.; Efros, L.; Caldwell, M.; Esteves, V.; Nakanishi, K.; Experimentia 1988, 44, 230.

13. Bankova, V. S.; Dyulgerov, A.; Popov, S. S.; Evstatieva, L.; Kuleva, L.; Pureb, O.; Zamjansan, Z.; Apidologie 1992, 23, 79.

14. Aga, H.; Shibuya, T.; Sugimoto, T.; Kurimoto, M.; Nakajima, S.; Biosci., Biotechnol., Biochem. 1994, 58, 945.

15. Ito, J.; Chang, F.-R.; Wang, H-K.; Park, Y. K.; Ikegari, M.; Kilgore, N.; Lee, K.-H.; J. Nat. Prod. 2001, 64, 1278.

16. Mahato, S. B.; Nandy, A. K.; Roy, G.; Phytochemistry 1992, 31, 2199.

17. Akihisa, T.; Yasukawa, K.; Oinuma, Hirotoshi; Kasahara, Y.; Yamanouchi, S.; Takido, Michio; Kumaki, K.; Tamura, T.; Phytochemistry 1996, 43, 1255 .

18. Silva, M. S. S.; Dissertação de Mestrado, Universidade Federal do Piauí, Brasil, 2004; Soares, J. D. M.; Coutinho, L. S.; Citó, A. M. G. L.; Lopes, J. A. D.; Chaves, M. H.; Oliveira, E. H.; Resumos da 24 Reunião Anual da Sociedade Brasileira de Química, Poços de Caldas, Brasil, 2001; Silva, M. S. S.; Citó, A. M. G. L.; Chaves, M. H.; Lopes, J. A. D.; Sousa, D. C.; Congresso Brasileiro de Química, Ouro Preto, Brasil, 2003

19. Singh, C.; Dev, S.; Tetrahedron 1977, 33, 817.

20. Wagner, H.; Bladit, S.; Zgainsky, E. M.; Plant drug analysis: A thin layer chromatography Atlas, $2^{\text {nd }}$ ed., Springer: Berlin, 1994.

21. Cardellina II, J. H.; J. Nat. Prod. 1983, 46, 196.

22. Bankova, V. S.; Popov, S. S.; Marekov, N. L.; Phytochemistry 1989, 28 , 871

23. Corsano, S.; Mincione, E.; Tetrahedron Lett. 1965, 28, 2377.

24. Anjaneyulu, V.; Harischandra-Prassad, K.; Ravi, K.; Connolly, J. D.; Phytochemistry 1985, 24, 2359.

25. Anjaneyulu, V.; Ravi, K.; Harischandra-Prassad, K.; Connolly, J. D.; Phytochemistry 1989, 28, 1471.

26. Anjaneyulu, V.; Satyanarayana, P.; Viswanadham, K. N.; Jyothi, V. G.; Nageswara-Rao, K.; Radhika, P.; Phytochemistry 1999, 50, 1229.

27. Januário, A. H.; Silva, M. F. G. F.; Vieira, P. C.; Fernandes, J. B.; Phytochemistry 1992, 31, 1251.

28. Pauleti, P. M.; Araújo, A. R.; Bolzani, V. S.; Quim. Nova 2002, 25, 349

29. Lago, J. H. G.; Roque, N. F.; Phytochemistry 2002, 60, 329.

30. Silvestein, R. M.; Webster, F. X.; Identificação Espectrométrica de Compostos Orgânicos, $6^{\mathrm{a}}$ ed, Livros Técnicos e Científicos Editora S. A.: Rio de Janeiro, 2000.

31. Olea, R. S. G.; Roque, N. F.; Quim. Nova 1990, 13, 278.

32. Lago, J. H. G.; Roque, N. F.; Phytochemistry 2002, 60, 329.

33. Furlan, M.; Roque, N. F.; Wolter Filho, W.; Phytochemistry 1993, 32, 1519. 